\title{
The Design of Bulk Carrier Cargo Holds State Integrated Monitoring System
}

\author{
Gao Ru-jiang, Wang Dan-li, Zou Chun-ming ${ }^{*}$, Xu Yan-min, Chen Min, Jin Cheng \\ School of Navigation, Wuhan University of Technology, Wuhan, 430063, China \\ Corresponding author: Zou Chun-ming
}

\begin{abstract}
Most ship cargo hold Internal uses artificial watch or is unattended. Therefore, it is impossible to know the appropriate information of the cargo holds Internal timely and accurately. Cargo damage and ship accidents occurred frequently. Automation remote processing and monitoring alarm system for the bulk carrier is an important part of the marine automation. The system plays a significant role to guarantee the navigation safety for bulk carriers. The paper introduced the important parts of the integrated monitoring system, structural design, hardware configuration, various modules communication transmission and various data processing software design were included. Based on embedded development, the real time information including the cargo hold internal humidity temperature, oxygen concentration, smoke gas concentration, cold water well level and other data were collected, as well the hatch closed state was detected and the cargo hold internal real time video information was collected. Then the real-time communication between the control display and acquisition modules were assured. By adjusting the corresponding buttons on the bridge according to the monitoring information, so that the cargo hold always in a safe environment, so as to avoid cargo hold accidents.
\end{abstract}

\section{Introduction}

With the rapid development of industry, science and technology, the automation ${ }^{[1]}$ of ship equipment and systems continues to increase. Ship centralized monitoring and alarm systems 错愄!未找到引用源。 as an important part of the ship automation, it is necessary to guarantee the safe navigation of the ship. In the world shipping market, bulk shipping accounted for about 30 percent of total global volume of bulk cargo shipping 琾: 未找到引用源。 have large carrying capacity, types of cargo transportation complex characteristics, especially the ship transports grains, feed and cargoes, It is very important to monitor the cargo hold temperature, humidity, oxygen concentration, cold water well level and other status information, and also very necessary to get the overall status of the cargo hold and cabin hatch real-time video information. Currently, in the whole bulk shipping industry, having little research on the status of the cargo cover and cabin hatch monitoring, and now most marine detectors are using foreign products including cabin leak detectors, leak detectors, water tightness detectors, and these products are only covered diagnosis for cargo covers but no cabin condition monitoring, and can not effectively predict the alarm, even can not realize remote manual 
or automatic processing. Therefore, for bulk cargo ship status monitoring study still in the relatively backward level. There are many accidents including cargo damage, wrecks and other accidents have been happened just because of having not comprehensive and timely understanding the cargo status information. Such as the "ZHE Chang-xing 6266" cargo ship aground accident on the September 7, 2014. It was due to careless crew forgot to close the inlet valves while loading coal, then a large salt water poured into the cabin, causing ship rapidly sunk. If the cargo status could be got in advance, then the accident would be avoided and reduced the loss of the ship and cargo. Thus, with the rapid development of marine transport economy, the importance of studying bulk cargo hold state comprehensive monitoring has become increasingly prominent.

\section{System design ideas}

With the large-scale promotion and application of computers embedded development, ship automation system equipment is also rising, but the ship automation on liquid cargo tanks错误!未找到引用源。 and ship engine room错误!未找到引用源。 are applied more than bulk cargo ships. By bring ship tanks and cabin condition monitoring into the ship's cargo hold, using the STM32 错误!未找到引用源。 and DSP 错误! 未找到引用源。 series of microcontrollers to develop, gathering the cargo temperature and humidity degrees, oxygen concentration, fire and smoke gas concentration, cold wells level and other status information, and by way of wired communications of $485 \mathrm{MHZ}$ and $2.4 \mathrm{G}$ wireless communications to achieve real-time data transmission. At last, the monitoring and early warning and automatic processing are achieved by the remote video information on the bridge.

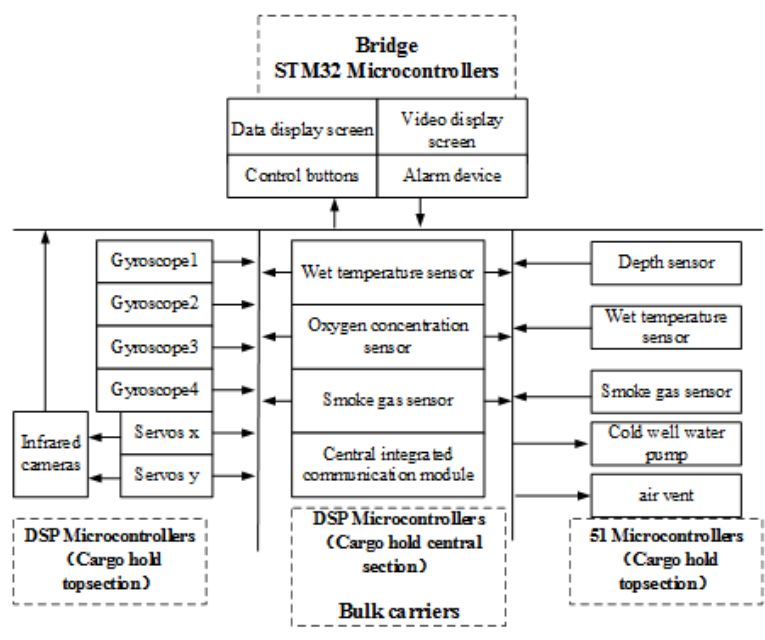

Figure 1. System Diagram

Seen from Figure 1, the system is composed of bridge controls display section, cabin top acquisition control section, central communication acquisition, side acquisition control section. System is based on STM32, DSP and 51 Microcontrollers to develop, by reasonably distributing cabin sensors including temperature sensor, humidity sensor, oxygen concentration sensor and other sensors to make up an embedded unit. The embedded modules are constituted based on data acquisition and processing and Microcontrollers performance computation. Each module between Lower Machine of the inner cabin uses $2.4 \mathrm{G}$ wireless communication method for data transmission, the central module belongs to the host part of the cargo is responsible for two-way data transmission of the top module and the side module and the control display communication section of the Position Machine. The RS-485 错误!未找到引用源。 serial bus standard communication mode was used on the cargo central module and bridge control display section, the standard mode uses balanced transmit and differential receive to suppress common mode interference. By using the principle of the three communications, the monitoring and control for real-time cargo was achieved well on the whole system. System top module, side and central module and display control module, see Figs. 2 and 3. 


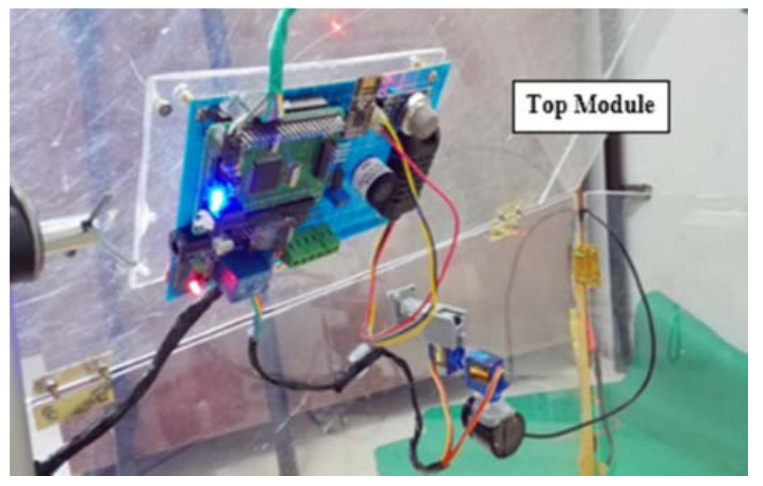

Figure 2. system top module

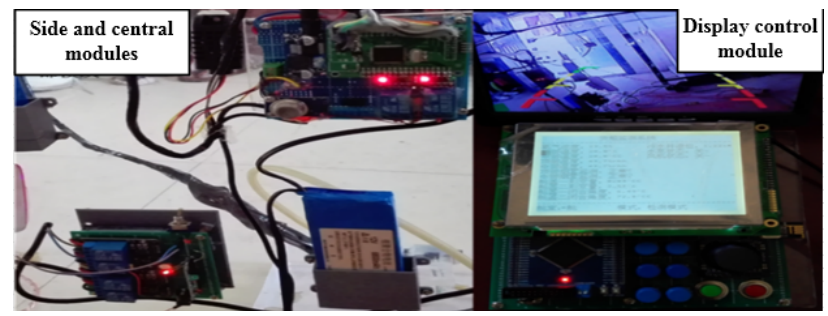

Figure 3. system side and central modules and display control module

\section{System research methods}

This paper uses system modularity step by step

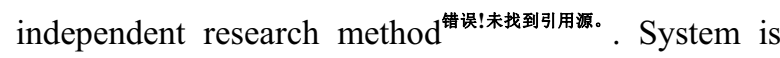
made up bridge control display section, cargo hold central monitoring section, cargo hold top detection section, cargo hold side section. The system is divided into hardware acquisition and transmission part and software calculation aspects. Here's what the work process to achieve hardware system development and PC software calculation process.

The hardware designs mainly related to the system power supply, pins link of communication module, anti-jamming circuit. PCA $82 \mathrm{C} 250$ is $5 \mathrm{~V}$ power supply chip, by using the CPLD to make level conversion for CANTX and CANRX, then $5 \mathrm{~V}$ will be converted to $3.3 \mathrm{~V}$ accepted by DSP. Since the ships are used in the engine room automation systems, the anti-jamming capability was enhanced.

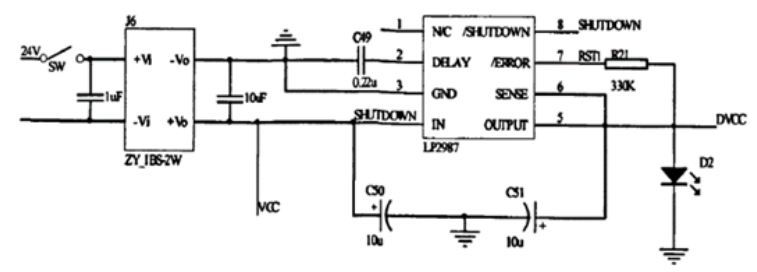

Figure 4. Power systems
The system uses a standard RS-485 serial bus communication mode, shown in Figure 4. The voltage and impedance are defined within the transmission processing, but the software protocols not. Different from RS232, RS485 features include:

(1) RS-485 electrical characteristics: logic "1" means a voltage difference between the two lines $+(2$ 6) V, logical "0" means the voltage difference between the two lines $-(2 \sim 6) \mathrm{V}$. The interface signal level lower than RS-232-C, therefore, it is not easy to damage in the chip interface circuit, and the level compatible with TTL level, can be easily connected with TTL circuits.

(2) The maximum data transfer rate of RS-485 is up to $10 \mathrm{Mbps}$.

(3) RS-485 interface is a combination of balanced drivers and differential receivers, enhanced resistance to common mode interference, which has a good noise immunity.

(4) The maximum standard transmission distance of RS-485 interface is 4000 feet (about 1219 meters), in fact, it can be up to 3000 meters, in addition, RS-232-C interface on the bus only allowed to connect just one transceiver, that is a single station ability. However, RS-485 interface on the bus is allowed to connect up to 128 transceivers. That is a multi-station capability, so users can take advantage of a single RS-485 interface to easily set up the device network. RS-485 can be constituted distributed systems by network. The "nodes" RS-485 is mainly according to the "receiver input impedance".

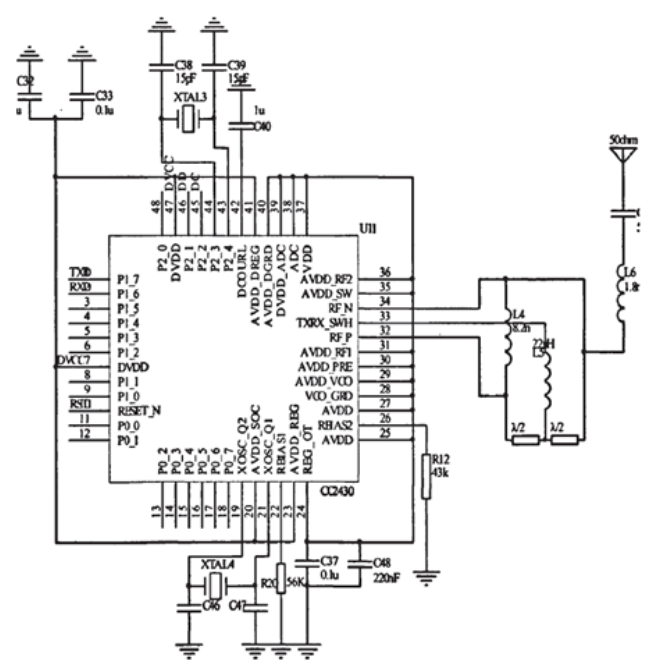

Figure 5.System communication pins configuration 
System simultaneously uses the temperature and humidity sensors, oxygen concentration sensors, fire and gas sensors, ultrasonic sensors, gyroscopes. For the sensor data acquisition and processing affect the microcontroller CPU resources. Therefore, the sensor should be reasonable pins configuration. see Figs 5 and 6.

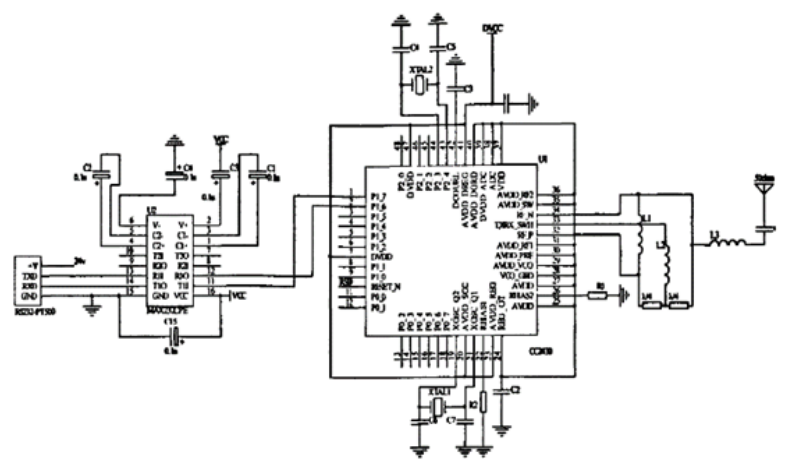

Figure 6.Sensor nodes configuration diagram

To sum up, the system power supply module, communication module, sensor configuration and anti-jamming circuit design are meet system requirements for real-time data acquisition and transmission, the system can be very good for a variety of real-time status information within the cargo hold monitoring and control. Stable and reliable hardware foundation provides a guarantee for the executing operation of software system.

\section{System software design and implementation}

Software design should be based on existing hardware resources and faced to applications requirements, to maximize improve the system functions. The design of the software system meets the requirements that the system collects many kinds of sensor data, real-time transmission requirement is high. The design of the software system makes monitoring to the network communication function as the center,

find the abnormal state quantity. When it is in monitoring data setting mode, it can set alarm threshold value for each state data by manually operating buttons, as shown in Figure 8 . both meet stable and reliable monitoring to the level of the cargo hold cold water wells, temperature and humidity, oxygen concentration and liquid fire gas concentration and other important parameters, but also to the achievement for smart combination of wired control network and sensor networks, as a result, both reliability and flexibility in two areas complement each other.

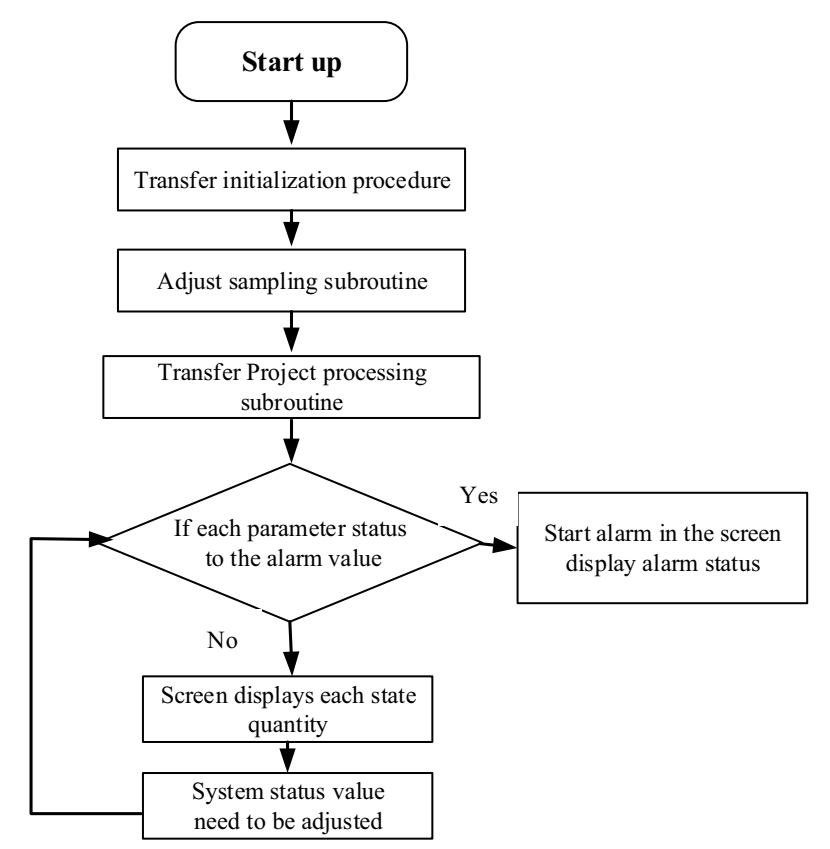

Figure 7. Software work flow chart

The monitoring software of control display section is a major component over the control layer. Control display section uses STM32F105VC model to develop, the display section in two modes, namely monitoring data display mode and monitor data setting mode. Different modes via a button on the control panel to switch. The system will display real-time status information when it is in monitoring data display mode. When there is abnormal situation occurs, the abnormal data will be marked, the operator can easily
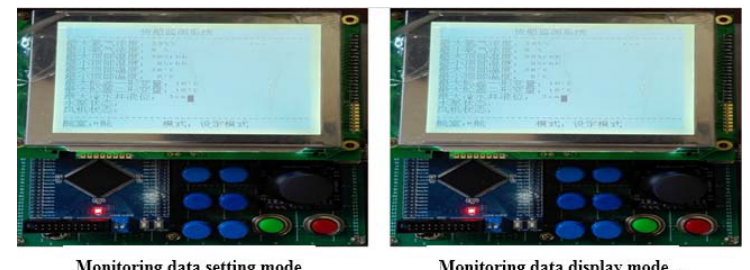

Monitoring data display mode

Figure 8. Two modes of Position Machine 
The software of system control display module can easily get transmission data, and clearly displayed on the screen. The module uses $3.3 \mathrm{~V}$ power supply, LCD high-brightness display, acrylic plastic spring button to ensure work reliability. In the Control Panel section, the cold well water pump switch within cargo hold and exhaust fan of the ventilation system can be manually controlled. By controlling cabin infrared camera shooting angles with joystick, the full range of real-time video cabin can be viewed well.

From the above analysis, the bulk cargo hold state monitoring system can connect and transport various of real-time information cabin. The data information is transmitted to bridge display section via RS-485 wired communication. Control display section has monitoring data display mode and monitoring data setting mode, operator can understand the real-time cabin temperature and humidity, oxygen concentration, the hatch closed, cold water well level and other status

\section{References}

1. Li Hong, Liu Yun, Lv Jian, Chen Yajie, Li Wenjie, $\mathrm{Xu}$ Bailing. Research and Application of the ship's engine room automation system generation software J diesel, 04 (2010): 21-23 + 33 .

2. Zhang shengtao. Study on the modern ship monitoring and alarm systems D Instructor: Sun Jianbo. Dalian Maritime University, (2010).

3. Wang Yunlong, Ji Zhuoshang, Lin Yan. Bulk carrier status and the development trend J Ship Engineering, 01 (2006): 58-61.

4. Wu Wenbin. Design and Implementation of tanker cargo hold monitoring system software J Ship and Ocean Engineering, 04 (2010): 82-83 + 87.

5. Zhao Xiaobian. Application of liquid level gauging in the monitoring of ship loading and unloading safety aspects D. instructor: Du Jiali. Dalian Maritime University, (2013).

6. Cao Yujiao. Study on ship engine room automatic monitoring system J. China Water Transport (next fortnightly), 12(2010): 107-109.

7. Zong Yang, Wang Jianhua. Design of engine room automation monitoring system based on wireless Ethernet J East China Shipbuilding Institute (Natural Science), 01 (2004): 27-31.

8. Wang Lijie, Xu Dekai. Research on hydraulic disc brakes intelligent monitoring system based STM32 MCU J Manufacturing Automation, 01 (2013: 48-49+ 58 .

9. Huang Peng, Yang Tianyi, Shi Weiren, Zhang Yuantao, Yin Lingling. Research on Marine information, and can watch real-time video information cabin, and can set data alarm value in the environment of different goods to automatically monitor, the operator can manually or automatically control all cabin ventilation system exhaust fan and cold water well pumps. The entire system played a significant role in ensuring the safety of navigation.

\section{System prospects}

The main scope of application of the system is in the ship bulk cargo transportation, the logistics warehousing and management and other relevant aspects can also be widely applied. In the bulk cargo transport logistics warehousing will has a wide range of applications, and also corresponding industries has a big application market. Application to bulk shipping will greatly improve the safety of shipping process. It will bring great economic benefits in the ship operation safety and reduction of goods loss aspects.

Engine Room Automation CAN bus communication system based on DSP J Ship Engineering, 02 (2010): 51-54.

10. Qin Bingbing, Li Huawen, Qin Qin. The achievement of RS-485 serial bus and communication $\mathrm{J}$ mine warfare and ship protection, 02(2011): 37-41.

11. Yang Meiwen. Design and development of sluice monitoring system based on RS-485 bus D Instructor: $\mathrm{Xu}$ Zhixiang, Dalian University of Technology, (2006).

12. Qiu Yonghong, Wang Tingchang, Fan Jianhua, Guo Ming. Study on modular design standard system based on wireless communication system J PLA University of Technology (Natural Science),02 (2003): 11-16. 